\title{
MANIFOLDS THAT FAIL TO BE CO-DIMENSION 2 FIBRATORS NECESSARILY COVER THEMSELVES
}

\author{
YOUNG HO IM and YONGKUK KIM
}

(Received 17 July 2001; revised 18 January 2002)

Communicated by S. Gadde

\begin{abstract}
Let $N$ be a closed s-Hopfian $n$-manifold with residually finite, torsion free $\pi_{1}(N)$ and finite $H_{1}(N)$. Suppose that either $\pi_{k}(N)$ is finitely generated for all $k \geq 2$, or $\pi_{k}(N) \cong 0$ for $1<k<n-1$, or $n \leq 4$. We show that if $N$ fails to be a co-dimension 2 fibrator, then $N$ cyclically covers itself, up to homotopy type.

2000 Mathematics subject classification: primary 57N15, 55M25; secondary 57M10, 54B 15. Keywords and phrases: residually finite group; s-Hopfian manifold; approximate fibration.
\end{abstract}

\section{Introduction}

The advantage of approximate fibration is that on one hand there exists an exact homotopy sequence but on the other hand there are more such approximate fibrations available. (See [3-5] for the definition and usefulness of approximate fibrations.)

To detect approximate fibrations, Daverman introduced the concept of co-dimension 2 fibrator as follows [7].

A closed $n$-manifold $N^{n}$ is a co-dimension 2 fibrator (respectively, a co-dimension 2 orientable fibrator) if, whenever $p: M \rightarrow B$ is a proper map from an arbitrary (respectively, orientable) $(n+2)$-manifold $M$ to a 2-manifold $B$ such that each $p^{-1}(b)$ is shape equivalent to $N$, then $p: M \rightarrow B$ is an approximate fibration.

All closed s-Hopfian manifolds with either trivial fundamental group or Hopfian fundamental group and nonzero Euler characteristic or hyper-Hopfian fundamental group are known to be co-dimension 2 fibrators $[6,7,16,18,19]$.

The first author was supported by grant No. R05-2000-000-00009-0 from the Basic Research Program of the Korea Science and Engineering Foundation.

The second author was supported by Kyungpook National University Research Fund, 2000.

(C) 2003 Australian Mathematical Society 1446-7887/03 \$A2.00+0.00 
For the sake of simplicity, we say that a closed manifold $N$ satisfies (CP) if $N$ cyclically covers itself nontrivially, up to homotopy type, and say that a closed manifold $N$ is (F) if $N$ fails to be a co-dimension 2 fibrator. Not only the torus and the Klein bottle but also $S^{1}$-bundles satisfy (CP).

It is well known [7, Theorem 4.2] that if a closed manifold $N$ satisfies (CP), then $N$ is (F). What can we say about the converse? Recently, Daverman [9] proves that the converse is not true in general, by showing that $S^{3} \times L(p, q)$ fails to be a co-dimension 2 fibrator but it cannot cover itself cyclically, where $L(p, q)$ is a Lens space.

It is natural to ask when the converse is true. A continuation of earlier investigations launched on [17], this paper adds evidence for a claim that the converse is true for many interesting manifolds. More precisely, we have the following

THEOREM. Suppose that a closed s-Hopfian n-manifold $N$ with residually finite, torsion free $\pi_{1}(N)$ and finite $H_{1}(N)$ is $(\mathbf{F})$. Then, $N$ satisfies $(\mathbf{C P})$, provided either

(1) $\pi_{k}(N)$ is finitely generated for all $k \geq 2$, or

(2) $\pi_{k}(N) \cong 0$ for $1<k<n-1$, in particular, aspherical manifold, or

(3) $n \leq 4$.

\section{Definitions and preliminaries}

Throughout this paper, the symbols $\simeq$ and $\cong$ denote a homotopy equivalence and an isomorphism, respectively. All manifolds are understood to be finite dimensional, connected and metric.

For a closed manifold $N$, a proper map $p: M \rightarrow B$ is $N$-like if each fiber $p^{-1}(b)$ is shape equivalent to $N$. For simplicity, we shall assume that each fiber $p^{-1}(b)$ in an $N$-like map to be an $A N R$ having the homotopy type of $N$.

Let $N$ and $N^{\prime}$ be (not necessarily closed) $n$-manifolds and $f: N \rightarrow N^{\prime}$ be a map. Denote the $k$ th cohomology group of $N$ with $\mathbb{G}$-coefficients and compact supports by $H_{C}^{k}(N ; \mathbb{G})$. If both $N$ and $N^{\prime}$ are orientable, then the degree of $f$ is the nonnegative integer $d$ such that the induced endomorphism $f^{*}: H_{C}^{n}(N ; \mathbb{Z}) \cong \mathbb{Z} \rightarrow H_{C}^{n}\left(N^{\prime} ; \mathbb{Z}\right) \cong \mathbb{Z}$ amounts to multiplication by $d$, up to sign. In general, the degree $\bmod 2$ of $f$ is the integer $d \in\{0,1\}$ such that the induced endomorphism $f^{*}: H_{C}^{n}\left(N ; \mathbb{Z}_{2}\right) \cong \mathbb{Z}_{2} \rightarrow$ $H_{C}^{n}\left(N^{\prime} ; \mathbb{Z}_{2}\right) \cong \mathbb{Z}_{2}$ amounts to multiplication by $d$.

Suppose that $N$ is a closed $n$-manifold and a proper map $p: M \rightarrow B$ is $N$-like. Let $G$ be the set of all fibers, that is, $G=\left\{p^{-1}(b): b \in B\right\}$. Put $C=\{p(g) \in B: g \in G$ and there exist a neighbourhood $U_{g}$ of $g$ in $M$ and a retraction $R_{g}: U_{g} \rightarrow g$ such that $R_{g} \mid g^{\prime}: g^{\prime} \rightarrow g$ is a degree one map for all $g^{\prime} \in G$ in $\left.U_{g}\right\}$, and $C^{\prime}=\{p(g) \in B: g \in$ $G$ and there exist a neighbourhood $U_{g}$ of $g$ in $M$ and a retraction $R_{g}: U_{g} \rightarrow g$ such 
that $R_{g} \mid g^{\prime}: g^{\prime} \rightarrow g$ is a degree one $\bmod 2$ map for all $g^{\prime} \in G$ in $\left.U_{g}\right\}$. Call $C$ the continuity set of $p$ and $C^{\prime}$ the mod 2 continuity set of $p$. Coram and Duvall showed [5] that $C$ and $C^{\prime}$ are dense, open subsets of $B$.

Call a closed manifold $N$ Hopfian if it is orientable and every degree one map $N \rightarrow N$ which induces a $\pi_{1}$-isomorphism is a homotopy equivalence. A closed manifold $N$ is $s$-Hopfian if $N$ is Hopfian when $N$ is orientable and $N_{H}$ is Hopfian when $N$ is non-orientable, where $N_{H}$ is the covering space of $N$ corresponding to $H=\bigcap_{i \in I}\left\{H_{i}:\left[\pi_{1}(N): H_{i}\right]=2\right\}$. By Hall's Theorem (for any finitely generated group $G$, the number of subgroups of $G$ having any fixed finite index is finite), the index set $I$ is finite, and so $H$ has a finite index in $\pi_{1}(N)$. All closed manifolds with virtually nilpotent or finite fundamental group, all closed aspherical manifolds, and all closed $n$-manifolds ( $n \leq 4)$ are examples of s-Hopfian manifolds. Whether all closed manifolds are s-Hopfian is related to the famous old problem of Hopf [14].

A group $\Gamma$ is said to be Hopfian if every epimorphism $f: \Gamma \rightarrow \Gamma$ is necessarily an isomorphism. A finitely presented group $\Gamma$ is said to be hyper-Hopfian if every homomorphism $f: \Gamma \rightarrow \Gamma$ with $f(\Gamma)$ normal and $\Gamma / f(\Gamma)$ cyclic is an isomorphism (onto). A group $\Gamma$ is said to be residually finite if for any non-trivial element $x$ of $\Gamma$ there is a homomorphism $f$ from $\Gamma$ onto a finite group $K$ such that $f(x) \neq 1_{K}$. It is well known that every finitely generated residually finite group is Hopfian.

Given a group $\Gamma$, we use $\Gamma^{\prime}$ to denote its commutator subgroup.

PROPOSITION 2.1 ([15] or [10]). Let $\psi: \Gamma \rightarrow \Gamma$ be an endomorphism of a finitely generated, residually finite group $\Gamma$ with $\Gamma^{\prime} \subset \psi(\Gamma)$. Then there exists an integer $k \geq 0$ for which $\psi$ restricts to a monomorphism on $\psi^{k}(\Gamma)$. Moreover, if $\Gamma / \Gamma^{\prime}$ is finite, then $\mathrm{ker} \psi$ is finite.

The next proposition is an easy consequence of the work of Epstein [13].

Proposition 2.2 ([2, Lemma 3.2]). Let $M$ and $N$ be manifolds and $f: M \rightarrow N a$ proper map such that $f_{\#}: \pi_{1}(M) \rightarrow \pi_{1}(N)$ is an isomorphism. Let $q^{\prime}: N^{\prime} \rightarrow N$ and $q^{\prime \prime}: M^{\prime \prime} \rightarrow M$ be coverings such that $q_{\#}^{\prime \prime}\left(\pi_{1}\left(M^{\prime \prime}\right)\right)=f^{-1}\left(q_{\#}\left(\pi_{1}\left(N^{\prime}\right)\right)\right)$. Suppose that $f^{\prime}: M^{\prime \prime} \rightarrow N^{\prime}$ is a lifting of $f \circ q^{\prime \prime}$ with $f \circ q^{\prime \prime}=f^{\prime} \circ q^{\prime}$. Then $\operatorname{deg} f=\operatorname{deg} f^{\prime} \in \mathbb{Z}_{2}$. Moreover, if $M$ and $N$ are orientable, then $\operatorname{deg} f=\operatorname{deg} f^{\prime} \in \mathbb{Z}$.

The following is basic for investigating co-dimension 2 fibrators.

LEMMA 2.3. Let $N$ be a closed $s$-Hopfian $n$-manifold with Hopfian fundamental group. If $N$ is (F), then at least one of the following two cases occurs:

Case 1: There is an $N$-like proper map $p: M^{n+2} \rightarrow \mathbb{R}^{2}$ defined on an $(n+2)$ manifold $M$ which is an approximate fibration over $\mathbb{R}^{2} \backslash 0$, but not an an approximate fibration over $\mathbb{R}^{2}$, such that $p^{-1}(0)$ is a strong deformation retract of $p^{-1}\left(\mathbb{R}^{2}\right) \equiv$ (say) $L$ under a retraction $R: L \rightarrow p^{-1}(0)$. 
Case 2: There is an $N$-like proper map $p: M^{n+2} \rightarrow \overline{\mathbb{H}}$ defined on an $(n+2)$-manifold $M$ which is an approximate fibration over $\mathbb{H}$, but not an an approximate fibration over $\overline{\mathrm{H}}$, such that $p^{-1}(\mathbf{0})$ is a strong deformation retract of $p^{-1}(\overline{\mathrm{H}}) \equiv($ say) $L$ under a retraction $R: L \rightarrow p^{-1}(\mathbf{0})$ and for all $\mathbf{a} \in \partial \mathrm{H}, R \mid p^{-1}(\mathbf{a}): p^{-1}(\mathbf{a}) \rightarrow p^{-1}(\mathbf{0})$ is a homotopy equivalence, where $\overline{\mathbb{H}}=\left\{(x, y) \in \mathbb{R}^{2} \mid y \geq 0\right\}, \mathbb{H}=\left\{(x, y) \in \mathbb{R}^{2} \mid y>0\right\}$ and $\partial \mathrm{H}=\left\{(x, y) \in \mathbb{R}^{2} \mid y=0\right\}$.

In either case, $\left(R \mid p^{-1}(x)\right)_{\#}\left(\pi_{1}\left(p^{-1}(x)\right)\right) \neq \pi_{1}\left(p^{-1}(0)\right)$ for some $x(\neq 0) \in \mathbb{R}$.

ProOF. If a closed s-Hopfian $n$-manifold $N$ with Hopfian fundamental group is (F), there is an $N$-like proper map $p: M^{n+2} \rightarrow B$ defined on an $(n+2)$-manifold $M$ which is not an approximate fibration. Hence $p: M^{n+2} \rightarrow B$ is not an approximate fibration at $x$ for some $x \in B$. Here $x \in C^{\prime}$ or $x \in \partial B$.

For the case of $x \in C^{\prime}$, applying [16, Theorem 3.1] and [7, Proposition 2.8], we can localize the situation into Case 1. Applying [7, Proposition 2.8] for the case of $x \in \partial B$, we can localize the situation into Case 2. In either case, the Hopfian hypotheses on $N$ and $\pi_{1}(N)$ gives $\left(R \mid p^{-1}(x)\right)_{\#}\left(\pi_{1}\left(p^{-1}(x)\right)\right) \neq \pi_{1}\left(p^{-1}(0)\right)$ for some $x(\neq \mathbf{0}) \in \mathbb{R}$.

\section{Proof of Main Theorem}

Suppose that a closed s-Hopfian $n$-manifold $N$ with residually finite, torsion free $\pi_{1}(N)$ and finite $H_{1}(N)$ is (F). By Lemma 2.3, at least one of the two cases occurs. Since the method of the proof of Case 2 is basically same as Case 1 , we only prove Case 1.

Put $g=p^{-1}(x)$ and $g_{0}=p^{-1}(0)$. Take the covering $\alpha: L_{l} \rightarrow L \equiv p^{-1}\left(\mathbb{R}^{2}\right)$ corresponding to incl ${ }_{*}\left(\pi_{1}(g)\right)$. Take the covering $\beta: L_{I H} \rightarrow L_{I}$ corresponding to $H=\cap_{t}\left\{H_{t} \leq \pi_{1}\left(L_{I}\right):\left[\pi_{1}\left(L_{I}\right): H_{t}\right]=2\right\}$ and then take the universal covering $\gamma: \tilde{L} \rightarrow L_{I H}$. Consider the following commutative diagram.

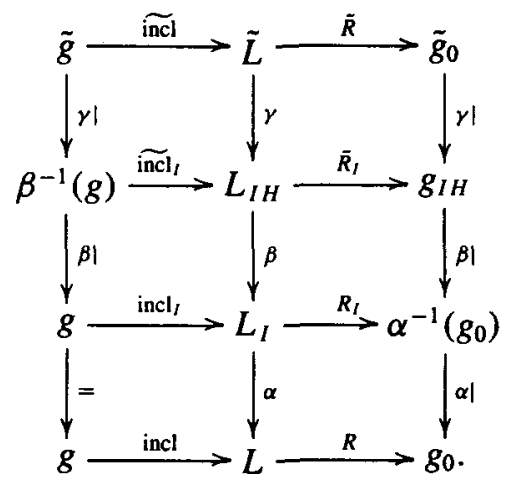


Here, incl $l_{l}$ and $R_{l}$ are liftings of the inclusion map incl and $R \circ q$, respectively. $\tilde{g}$ and $\tilde{g}_{0}$ are the universal covering of $N$.

First, we claim that $R_{l}$ o incl $l_{l}$ induces a $\pi_{1}$-isomorphism.

Since $p$ is an approximate fibration over $\mathbb{R}^{2} \backslash \mathbf{0}$, there is a homotopy exact sequence

$$
\pi_{1}(g) \rightarrow \pi_{1}\left(p^{-1}\left(\mathbb{R}^{2} \backslash \mathbf{0}\right)\right) \rightarrow \pi_{1}\left(\mathbb{R}^{2} \backslash \mathbf{0}\right) \cong \mathbb{Z} \rightarrow 1
$$

showing $\pi_{1}\left(p^{-1}\left(\mathbb{R}^{2} \backslash \mathbf{0}\right)\right) / \operatorname{incl}_{\#}\left(\pi_{1}(g)\right) \cong \mathbb{Z}$. Because $g$ has the homotopy type of a co-dimension 2 compactum from $L$, the inclusion $p^{-1}\left(\mathbb{R}^{2} \backslash \mathbf{0}\right) \rightarrow L$ induces an epimorphism $\varphi$ of fundamental groups. It follows directly that $R_{\sharp} \varphi \operatorname{incl}\left(\pi_{1}(g)\right)$ is a normal subgroup of $\pi_{1}\left(g_{0}\right)$ having cyclic cokernel. Hence, incl $\left(\pi_{1}(g)\right)$ contains the commutator subgroup $\pi_{1}(L)^{\prime}$ of $\pi_{1}(L)$. Since $\pi_{1}(L) \cong \pi_{1}\left(g_{0}\right) \cong \pi_{1}(g) \cong \pi_{1}(N)$ is residually finite and $\pi_{1}(N) / \pi_{1}(N)^{\prime} \cong H_{1}(N)$ is finite, by Proposition $2.1, \operatorname{ker}\left(\right.$ incl $\left._{\#}\right)$ is finite. But since $\pi_{1}(g)$ is torsion free, $\operatorname{ker}\left(\right.$ incl $\left._{\#}\right)$ should be trivial, that is, incl : : $\pi_{1}(g) \rightarrow \pi_{1}(L)$ is a monomorphism. Consequently, $\left(\operatorname{incl}_{I}\right)_{\|}: \pi_{1}(g) \rightarrow \pi_{1}\left(L_{l}\right)$ is an isomorphism, for $q_{*} \circ\left(\text { incl }_{I}\right)_{\#}=$ incl $_{*}$ and $q_{\#}$ is a monomorphism.

Since there is no upper semicontinuous decomposition of an orientable $(n+2)$ manifold consisting entirely of nonorientable $n$-manifolds [7, Proposition 2.9], the orientability of $L_{I H}$ implies the orientability of $\beta^{-1}(g)$. So by [11, Lemma 5.5], the index $\left[\pi_{1}\left(g_{I H}\right):\left(\tilde{R}_{I} \circ \text { incl }_{l}\right)_{*}\left(\pi_{1}\left(\beta^{-1}(g)\right)\right)\right]$ equals to the degree of the map $\tilde{R}_{I} \circ \widetilde{\text { incl }}_{I}$. Applying the fact [18, Lemma 3.2] that $R_{I} \circ$ incl $_{I}$ induces a $\pi_{1}$-isomorphism if and

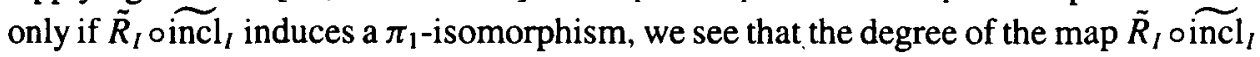
must be one.

(1) First assume that $\pi_{k}(N)$ is finitely generated for all $k \geqq 2$.

By Proposition 2.2, we have that the degree of the map $\tilde{R} \circ$ incl is one. Hence $\tilde{R} \circ \widetilde{\text { incl }}$ induces $H_{C}^{k}$-monomorphisms for all $k$. By the fact of $H_{C}^{k}(X ; \mathbb{G}) \cong H_{n-k}(X ; \mathbb{G})[21$,

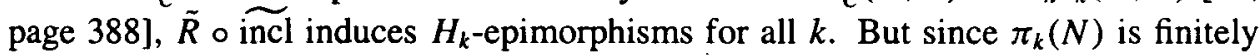
generated for all $k \geq 2, H_{k}(\tilde{g}) \cong H_{k}\left(\tilde{g}_{0}\right)$ is a finitely generated Abelian group [22, page 509] (and so it is Hopfian) so that $(\tilde{R} \circ \widetilde{\text { incl }})_{k}: H_{k}(\tilde{g}) \rightarrow H_{k}\left(\tilde{g}_{0}\right)$ is an isomorphism. Appealing to the Hurewicz Theorem, we see that $\pi_{k}(\tilde{g}) \rightarrow \pi_{k}(\tilde{L})$ is an isomorphism for all $k \geq 2$. Whitehead's Theorem ensures that the composition $g \rightarrow L_{l} \rightarrow \alpha^{-1}\left(g_{0}\right)$ is a homotopy equivalence. But since $\alpha \mid: \alpha^{-1}\left(g_{0}\right) \rightarrow g_{0}$ is a covering map, $g_{0} \simeq N$ satisfies (CP).

(2) Next, assume that $\pi_{k}(N) \cong 0$ for $1<k<n-1$.

Recall the work of Swarup [23]: For a map $f: A \rightarrow B$ between closed oriented $n$-manifolds with $\pi_{1}$-isomorphism and $\pi_{k}(A)=\pi_{k}(B)=0$ for $1<k<n-1, f$ is a homotopy equivalence if and only if $\operatorname{deg} f=1$.

Since the degree of the map $\tilde{R}_{I} \circ \widetilde{\text { incl }}_{I}$ is one, by the work of Swarup, $\tilde{R}_{I} \circ \widetilde{\text { incl }_{I}}$ is a homotopy equivalence.

(3) Finally, assume that $n \leq 4$.

The case of $n=3$ is a special case of (2). 
For the case of $n=4$, apply the following consequence of the work of Hausmann [14]: For any degree one map $f: A^{4} \rightarrow B^{4}$ between closed 4-manifolds with $\pi_{1}$-isomorphism, $f$ is a homotopy equivalence.

Although Hausmann only proves the case $A=B$, just mimicking his proof and using the exact sequence of surgery with Poincaré duality, one may deduce the statement above.

\section{Example and remarks}

EXAMPLE ([12]). A closed $n$-manifold $N, n>4$, which fails to be a co-dimension 2 fibrator but $H_{1}(N) \cong \mathbb{Z}_{2} \oplus \mathbb{Z}_{2}$ and every $\gamma \in \pi_{1}(N), \gamma \neq 1$, has infinite order.

Apply Maunder's construction [20] to obtain a finite aspherical 2-complex $K$ such that $H_{1}(K) \cong \mathbb{Z}_{2}$. Specify a PL embedding of $K$ in an $(n+1)$-manifold $M^{n+1}$, and let $S$ be the boundary of a regular neighbourhood of the image. Let $\Omega$ be the mapping cylinder of a 2-1 covering map $\Theta: \bar{S} \rightarrow S$; here $\Omega$ is a (non-orientable) twisted $I$-bundle over $S$. Form $N$ by doubling $\Omega$ along $\bar{S}$, its boundary.

A routine computation involving a Mayer-Vietoris sequence confirms $H_{1}(N) \cong$ $\mathbb{Z}_{2} \oplus \mathbb{Z}_{2}$. Note that $\pi_{1}(\Omega) \cong \pi_{1}(S) \cong \pi_{1}(K)$, from which it follows that $\pi_{1}(N) \cong$ $\pi_{1}(\Omega) *_{\pi_{1}(\bar{S})} \pi_{1}(\Omega)$ is the fundamental group of an aspherical finite complex and, hence, no nontrivial element has finite order [1, Corollary VIII.2.5].

Such manifolds $N$ fail to be co-dimension 2 fibrators, due to the existence of a 2-1 covering map $N \rightarrow N$ (see [7, Theorem 4.2]). For the most obvious 2-1 covering $\bar{N} \rightarrow N, \bar{N}$ will consist of two copies $\Omega_{1}, \Omega_{2}$ of $\Omega$, arising as the preimage of one $\Omega$ in the target space, $N$, together with a 2-1 covering $\bar{\Omega}$ of the other copy of $\Omega$ used to form $N$. But here $\bar{\Omega}$ is simply $\bar{S} \times[0,1]$, and $\bar{N}=\Omega_{1} \cup(\bar{S} \times[0,1]) \cup \Omega_{2}$ with attachments that reveal $\bar{N} \approx N$.

Note that for all $i \geq 2, \pi_{i}\left(N \times S^{k}\right)(k \geq 2)$ is finitely generated.

REMARK. The condition of torsion free $\pi_{1}(N)$ cannot be omitted, since $S^{3} \times L(p, q)$ fails to be a co-dimension 2 fibrator ([9]) but it cannot cover itself cyclically, where $L(p, q)$ is a Lens space.

On the other hand, the condition of finite $H_{1}(N)$ is also imperative, since $N$ fails to be a co-dimension 2 fibrator but it cannot cover itself cyclically, where $N$ is some Nil 3-manifold (See [8]).

\section{References}

[1] K. S. Brown, Cohomology of groups (Springer, New York, 1982). 
[2] N. Chinen, 'Products of manifolds with nonzero Euler characteristic and co-dimension 2 fibrators', preprint.

[3] D. S. Coram and P. F. Duvall, 'Approximate fibration', Rocky Mountain J. Math. 7 (1977), 275-288.

[4] _ 'Approximate fibration and a movability condition for maps', Pacific J. Math. 72 (1977), $41-56$.

[5] _ _ 'Mappings from $S^{3}$ to $S^{2}$ whose point inverses have the shape of a circle', General Topology Appl. 10 (1979), 239-246.

[6] R. J. Daverman, 'Hyper-Hopfian groups and approximate fibrations', Compositio Math. 86.

[7] — 'Submanifold decompositions that induce approximate fibrations', Topology Appl. 33 (1989), 173-184.

[8] _ _ '3-manifolds with geometric structure and approximate fibrations', Indiana Univ. Math. J. 40 (1991), 1451-1469.

[9] —_ 'Co-dimension 2 fibrators with finite fundamental groups', Proc. Amer. Math. Soc. 127 (1999), 881-888.

[10] — , 'Hyperbolic groups are hyper-Hopfian', J. Austral. Math. Soc. (Series A) 68 (2000), 126130.

[11] R. J. Daverman, Y. H. Im and Y. Kim, 'Products of Hopfian manifolds and co-dimension 2 fibrators', Topology Appl. 103 (2000), 323-338.

[12] R. J. Daverman and Y. Kim, '2-groups and approximate fibrations', Topology Appl. , to appear.

[13] D. B. A. Epstein, 'Degree of a map', Proc. London Math. Soc. 16 (1966), 369-383.

[14] J. C. Hausmann, Geometric Hopfian and non-Hopfian situations, Lecture Notes in Pure Appl. Math. (Marcel Decker, NY, 1987) pp. 157-166.

[15] R. Hirshon, 'Some properties of endomorphisms in residually finite groups', J. Austral. Math. Soc. (Series A) 24 (1977), 117-120.

[16] Y. H. Im and Y. Kim, 'Hopfian and strongly Hopfian manifolds', Fund. Math. 159 (1999), 127-134.

[17] — 'Necessary and sufficient conditions for s-Hopfian manifolds to be co-dimension 2 fibrators', Proc. Amer. Math. Soc. 129 (2001), 2135-2140.

[18] Y. Kim, 'Manifolds with hyper-Hopfian fundamental group as co-dimension 2 fibrators', Topology Appl. 96 (1999), 241-248.

[19] — 'Strongly Hopfian manifolds as co-dimension 2 fibrators', Topology Appl. 92 (1999), 237-245.

[20] C. R. F. Maunder, 'A short proof of a theorem of Kan and Thurston', Bull. London Math. Soc. 13 (1981), 325-327.

[21] J. R. Munkres, Elements of algebraic topology (Addison-Wesley, Menlo Park, CA, 1984).

[22] E. H. Spanier, Algebraic topology (Springer, New York, 1995).

[23] G. A. Swarup, 'On a theorem of C. B. Thomas', J. London Math. Soc. 8 (1974), 13-21.

Department of Mathematics

Pusan National University

Pusan 609-735

Korea

e-mail: yhim@pusan.ac.kr
Department of Mathematics Kyungpook National University Taegu 702-701

Korea

e-mail: yongkuk@knu.ac.kr 
